\title{
A Multicenter Evaluation of Chronic Ulcer Recurrence with the Use of Varying Mechanical Wound Healing Modalities
}

\section{Rosenblum $\mathrm{J}^{1 *}$, Gazes $\mathrm{MI}^{2}$, Rosenblum $\mathrm{S}^{1}$, Karpf $\mathrm{A}^{1}$ and Greenberg $\mathrm{N}^{3}$}

${ }^{1}$ Shaarei Zedek Medical Center, Jerusalem, Israel

${ }^{2}$ Yale New Haven Hospital, New Haven, CT, Israel

${ }^{3}$ Diabetic Foot Service, Department of Orthopedics, Shaarei Zedek Medical Center, Jerusalem, Israel

\begin{abstract}
Chronic wound care is a growing medical problem, reaching epidemic proportions worldwide. Even after the wound has healed, wound recurrence remains a significant issue, with rates approximating $40 \%$ in 6 months after healing. The authors evaluate a retrospective analysis of their wound care clinics healing and recurrence rates. The authors evaluate the efficacy of varying physical modalities on preventing recurrence of wounds after their healing The authors take their evaluation out to one year.
\end{abstract}

Keywords: Diabetic foot ulcer; Venous leg ulcer; Ultrasound; Wound recurrence

\section{Introduction}

Foot and leg ulcerations, depending on the individual person, heal at different rates. Certain wounds have simple healing routes without future recurrence while others last for an extended period of time or reoccur after initially succeeding with skin closure. In the cases where ulcerations fail to heal at a timely rate, infection may occur, leading to more serious complications which could cause significant loss of limb or in worst case scenarios, loss of life [1]. Underlying causes of ulcerations, including infections, co-morbidities, abnormal biomechanics, and individual lifestyle and behavior play large roles in determining the ultimate course of ulceration and recurrence [2-4]. Two such examples of ulcerations which frequently relapse include the diabetic foot ulcer and the venous leg ulcer. Diabetic foot ulcerations are an example of a type of wound which may take an extended period of time to heal. After healing, diabetic foot ulcers frequently reoccur. Peters et al. enrolled 81 subjects with ulcerations distal to the ankle into a cohort study evaluating risk factors for secondary diabetic foot ulcers [5]. The authors found that $12.3 \%$ of ulcerations did not heal by the end of their follow-up period. $16.0 \%$ of the patients progressed to a lower extremity amputation. The study concluded that peripheral vascular disease, repetitive injury to an insensate foot, and incorrectly fitted shoe gear increased risk for ulceration in the diabetic patient. Dubsky et al. examined data of patients who had relapses of diabetic foot ulcers and found that recurrence occurred in $57.5 \%$ of the patients in this population, with predictors for recurrence including plantar ulcer location, presence of osteomyelitis, C-Reactive Protein levels greater than $5 \mathrm{mg} / \mathrm{L}$, and Hemoglobin Alc greater than $7.5 \%$ [6]. Ghanassia et al. evaluated the long term outcomes for patients hospitalized for diabetic foot infection. In this population, $43.8 \%$ of patients underwent amputation while $51.7 \%$ died within a two year study period, albeit half of the mortality was from cardiovascular events. $60.9 \%$ of patients in this evaluation had ulcer recurrence [7]. Apelqvist et al. found reulceration rates up to $40 \%$ within 3 years and up to $70 \%$ within 5 years within a diabetic population [8]. Galea et al. determined a $48.5 \%$ ulcer recurrence in subjects included in their study, with all but 1 patient having type II diabetes mellitus [9]. In this evaluation, the average time duration of patients suffering from diabetes was 12.5 years. The most common re-ulceration regions were on the plantar foot in $43.8 \%$ of the subjects, the apex of the toes in $31.3 \%$, dorsally in $15.6 \%$, and on the lateral heel in $9.4 \%$. Different modalities and techniques have been utilized in attempts to prevent the diabetic foot from having ulceration recurrence. For example, Lavery et al. examined the use of temperature monitoring in a physician-blinded, randomized multicenter trial, enrolling patients with histories of diabetic foot ulcerations. One group within the study was provided with an infrared skin thermometer to measure temperatures on different areas of the foot. These subjects had less ulcerations than the study groups who were not provided with a thermometer, demonstrating that knowledge of skin temperature measurements assisted in recurrence rate reduction [10]. Venous leg ulcerations are also at high risks of recurrence after obtaining an initial skin closure. Venous leg ulcerations are the most common form of lower extremity ulcers in the United States, affecting about $1 \%$ of the population. Causes of the ulcerations include inflammation leading to leukocyte activation, platelet aggregation, damage to endothelium, and intracellular edema [11]. Obesity, deep vein thrombosis, history of leg injury, age (elderly), phlebitis, and sex (women) are the chief risk factors leading to venous stasis development and ulceration. Eczematous dermatologic changes, hyperpigmentation on surrounding skin, edema upon dependence, varicose veins, and dull pain are related to venous stasis recognition. Additionally, bony prominences typically underlie the ulceration. The venous hypertension leading to ulceration and recurrence can be decreased via non-invasive mechanisms including compression therapies, or if needed, surgical procedures [12]. McDaniel et al. reviewed recurrence risks based on "clinical, etiologic, anatomic, and pathophysiologic criteria and hemodynamic characteristics of the affected limb as assessed with air plethysmography [13]. Corrective venous surgical interventions were performed in 37 extremities of the 99 assessed in the study. Recurrence of venous leg ulcerations was approximately $37 \%$ at 3 years and about $48 \%$ at 5 years. Those who had surgical intervention for the venous leg ulceration had a recurrence rate of about $27 \%$ at 4 years, which was less than those undergoing

*Corresponding author: Jonathan Rosenblum, DPM, Shaarei Zedek Medical Center, Jerusalem, Israel, Tel: +972-50-595-5161; E-mail: diabfootman@gmail.com

Received June 05, 2017; Accepted June 26, 2017; Published June 29, 2017

Citation: Rosenblum J, Gazes MI, Rosenblum S, Karpf A, Greenberg N (2017) A Multicenter Evaluation of Chronic Ulcer Recurrence with the Use of Varying Mechanical Wound Healing Modalities. J Vasc Med Surg 5: 323. doi: 10.4172/23296925.1000323

Copyright: (c) 2017 Rosenblum J, et al. This is an open-access article distributed under the terms of the Creative Commons Attribution License, which permits unrestricted use, distribution, and reproduction in any medium, provided the original author and source are credited. 
non-invasive interventions. Deep venous insufficiency was found to have significantly higher recurrence rates. Reeder et al. performed a literature search on venous leg ulcer recurrence rates, noticing that recurrence ranges from $0 \%$ at 6 months to $56 \%$ at 4.5 years and voicing a need for new strategies in post venous leg ulceration patients [14]. This study evaluates both diabetic foot ulcerations and venous leg ulcerations treated with ultrasound and electrical stimulation therapy and the ulceration recurrence rate post intervention.

\section{Methods}

This was a retrospective analysis of patients attending clinics between 2008 and 2014. Patients were treated for either diabetic or venous lesions. Patients had suffered from am ulceration for a minimum of 2 months prior to healing. Patients were enrolled in the study, only if they fit into only one of the treatment protocols: Advanced Wound Care Products (AWC), Conventional Ultrasound (1-3 mHz), Conventional Electric Stimulation, Surface Acoustic Wave Therapy (SAW) (NanoVibronix Inc. Elmsford, NY, USA), Combined Ultrasound and Electric Field Stimulation (CUSEFS) (BRH Medical, Jerusalem, Israel), and Ultrasonic Debridement. If a patient received more than one category of treatment other than having AWC in addition to a mechanical modality, in order to be included in the study, patients had to attend at least $60 \%$ of their follow up visits. These centers all utilized the same post healing treatment and prevention regimen which included for DFU's: quarterly visits, specialized shoegear and or thoses, routine foot care including moisturizing as well as daily home foot exams. For VLU's the treatment regimen included using compression therapy, either a bandage or stocking from toe to knee, as well as routine skin care with a moisturizer. Binary clinical outcomes were collected at 6 and 12 months following treatment. The binary outcome was an assessment of whether the lesion had recurred. Patients with recurring lesions were marked with a 1 and patients with no recurring lesions were marked with a zero. Thus, for each of the 6 treatment groups, the outcome variable of interest was the percent of recurring lesions at 6 and 12 months. Diabetic and venous lesions were combined at each assessment point. Thus, at each point of assessment, 6 treatment conditions were compared to each other in terms of whether they differ in the overall percent of both diabetic and venous recurring lesions Logistic regression was utilized to model the binary outcomes data. Because the AWS condition is considered the standard of care it was made the reference group against which all other groups were compared. Following the initial logistic regression, any group that was statistically different from the AWS standard of care was then compared to all other groups. These analyses were carried out for separately at the 6 and 12 month points of assessment.

\section{Results}

The percent of recurring lesions for each of the 6 groups at 6 and 12 month assessment points are presented in Figure 1. Tables 1 and 2 show the data sets reported at both 6 and 12 months. At 6 months, logistic regression showed that the SAW and BRH conditions had significantly fewer recurring lesions compared to the AWS standard of care condition (SAW: $\mathrm{B}=2.1, \mathrm{OR}=7.9$, Wald=7.7, $\mathrm{p}<.001$; $\mathrm{BRH}$ : $\mathrm{B}=2.9, \mathrm{OR}=17.4$, Wald=15.1, $\mathrm{p}<.001$ ). The Odds Ratios and $\mathrm{p}$ values comparing SAW and AWS with all other conditions are presented in Tables 3 and 4. SAW had significantly fewer lesions compared to all other conditions except US. BRH had significantly fewer lesions compared to all other conditions. SAW and BRH did not differ from each other (SAW: $\mathrm{B}=.8, \mathrm{OR}=.45$, Wald=.6, $\mathrm{p}=.45$ ) At 12 months, logistic regression showed that the SAW and BRH conditions had

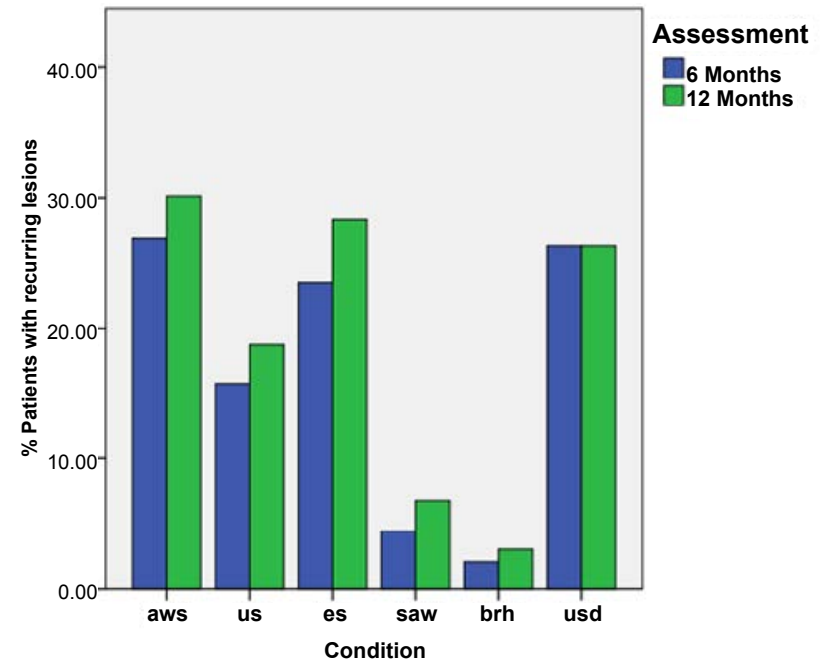

Figure 1: Overall Results of the Study.

significantly fewer recurring lesions compared to the AWS standard of care condition (SAW: $\mathrm{B}=1.8, \mathrm{OR}=5.9$, Wald $=8.1, \mathrm{p}<. .05 ; \mathrm{BRH}$ : $\mathrm{B}=2.6, \mathrm{OR}=13.5$, Wald $=18.2, \mathrm{p}<.001$ ). The Odds Ratios and $\mathrm{p}$ values comparing SAW and AWS with all other conditions are presented in Table 3. SAW had significantly fewer lesions compared to all other conditions except US. BRH had significantly fewer lesions compared to all other conditions. SAW and BRH did not differ from each other (SAW: $\mathrm{B}=.83, \mathrm{OR}=.44$, Wald $=.98, \mathrm{p}=32$ ).

\section{Discussion}

Ultrasound application has the ability to alter blood flow, angiogenesis, cellular protein synthesis, vascular permeability, and collagen make-up and configuration [15]. Yao et al. determined that the use of ultrasound promoted a reduction in pro-inflammatory cytokines and enhancement of tissue regeneration [16]. The study evaluated nonhealing diabetic foot ulcerations in patients with neuropathy treated via ultrasound for 5 weeks. The group who used ultrasound at increased intervals throughout the treatment period had significant wound area reduction. Ultrasound was deemed effective in treating chronic neuropathic diabetic foot ulcers. Electrical stimulation therapy affects the electrochemical wound process. Intact skin has a transepithelial potential, with the skin surface having negative chloride ions and the dermis having positive sodium ions. Ulcerations have abnormal potentials which promote wound healing. The potential decreases in chronic wounds, leading to less healing potential. Electrical stimulation reestablishes the currents found in the transepithelial potential and stimulates the healing process [17]. Peters et al. evaluated high voltage, pulse galvanic electrical stimulation on diabetic ulcerations, noticing increased healing in $65 \%$ of patients compared to a control group with no electrical stimulation [18]. Similarly, Baker et al. examined electrical stimulation therapy waveforms on diabetic ulceration healing rates, determining that patients have significantly increased healing rates of nearly $60 \%$ when utilizing asymmetric biphasic waveforms in comparison to a control group with no electrical stimulation therapy [19]. Ultrasound and electrical stimulation have also been shown to treat venous leg ulcerations. Taradaj et al. examined 81 patients treated either with surgery or less invasive measures, including therapeutic ultrasound. The study concluded that ultrasound is useful and efficient as a non-invasive measure for venous leg ulcerations [20]. Samuels et al. noticed that ultrasound promoted wound closure by leading to 
Citation: Rosenblum J, Gazes MI, Rosenblum S, Karpf A, Greenberg N (2017) A Multicenter Evaluation of Chronic Ulcer Recurrence with the Use of Varying Mechanical Wound Healing Modalities. J Vasc Med Surg 5: 323. doi: 10.4172/2329-6925.1000323

Page 3 of 4

\begin{tabular}{|c|c|c|c|c|c|c|}
\hline & $\begin{array}{c}\text { Advanced Wound } \\
\text { Care Products }\end{array}$ & Ultrasound & Electric Stimulation & $\begin{array}{c}\text { Surface } \\
\text { Acoustic Waves }\end{array}$ & $\begin{array}{c}\text { BRH Combined } \\
\text { Ultrasound and Electric } \\
\text { Field Stimulation }\end{array}$ & $\begin{array}{c}\text { Ultrasound Assisted } \\
\text { Debridement }\end{array}$ \\
\hline $\begin{array}{c}\text { Number of Cases Total at 6 } \\
\text { months }\end{array}$ & 175 & 51 & 64 & 45 & 97 & 38 \\
\hline Venous(V)/ Diabetic(D) at 6 months & $64 / 111$ & $21 / 30$ & $24 / 40$ & $12 / 33$ & $43 / 54$ & $9 / 29$ \\
\hline Recurrence at 6 months V/D & $17 / 30$ & $3 / 5$ & $6 / 9$ & $1 / 1$ & $1 / 1$ & $2 / 8$ \\
\hline \% Recurrence at 6 months V/D & $26.6 / 27.0$ & $14.29 / 16.67$ & $25 / 22.5$ & $8.3 / 3.03$ & $2.33 / 1.85$ & $29.0 / 27.59$ \\
\hline Total Recurrence at 6 months & 47 & 8 & 15 & 2 & 2 & 10 \\
\hline Total \% recurrence at 6 months & 26.8 & 15.7 & 26.4 & 4.4 & 2.06 & 26.3 \\
\hline
\end{tabular}

Table 1: Data set at 6 months.

\begin{tabular}{|c|c|c|c|c|c|c|}
\hline & $\begin{array}{l}\text { Advanced Wound } \\
\text { Care Products }\end{array}$ & Ultrasound & Electric Stimulation & $\begin{array}{c}\text { Surface } \\
\text { Acoustic Waves }\end{array}$ & $\begin{array}{l}\text { BRH Combined } \\
\text { Ultrasound and Electric } \\
\text { Field Stimulation }\end{array}$ & $\begin{array}{c}\text { Ultrasound Assisted } \\
\text { Debridement }\end{array}$ \\
\hline $\begin{array}{c}\text { Number of Cases Total at } 12 \\
\text { months }\end{array}$ & 166 & 48 & 60 & 44 & 97 & 38 \\
\hline Venous/ Diabetic at 12 months & $61 / 105$ & $20 / 28$ & $23 / 37$ & $12 / 32$ & $43 / 54$ & $9 / 29$ \\
\hline Recurrence at 12 months V/D & $19 / 31$ & $4 / 5$ & $7 / 10$ & $1 / 2$ & $2 / 2$ & $2 / 8$ \\
\hline$\%$ Recurrence at 12 months V/D & $31.1 / 31$ & $20 / 17.9$ & $30.4 / 27.03$ & $8.3 / 6.25$ & $4.65 / 3.70$ & $29.0 / 27.59$ \\
\hline Total Recurrence at 12 months & 50 & 9 & 17 & 3 & 4 & 10 \\
\hline Total \% recurrence at 12 months & 30.12 & 18.75 & 28.33 & 6.81 & 4.12 & 26.3 \\
\hline
\end{tabular}

Table 2: Data set at 12 months.

\begin{tabular}{|c|c|c|c|c|}
\hline & AWC & US & ES & USD \\
\hline SAW & $7.9^{*}$ & 4 & $6.5^{*}$ & $7.7^{*}$ \\
\hline BRH & $17.4^{* *}$ & $8.8^{* *}$ & $14.5^{* *}$ & $17^{* *}$ \\
\hline
\end{tabular}

${ }^{*} \mathrm{p}<.05 ;{ }^{* *} \mathrm{p}<.001$

Table 3: Odds Ratios for SAW and BRH compared to all other conditions at 6 months.

\begin{tabular}{|c|c|c|c|c|}
\hline & AWC & US & ES & USD \\
\hline SAW & $5.9^{*}$ & 3.2 & $5.4^{*}$ & $4.9^{*}$ \\
\hline BRH & $13.5^{* *}$ & $7.2^{* *}$ & $12.4^{* *}$ & $11.2^{* *}$ \\
\hline
\end{tabular}

${ }^{*} p<.05 ;{ }^{* *} p<.001$

Table 4: Odds Ratios for SAW and BRH compared to all other conditions at 12 months.

proliferation and cellular metabolism [21]. Junger et al. evaluated the economic and therapeutic results of electrical stimulation on venous leg ulcerations [22]. The authors noted that pain level, ulceration measurements, transcutaneous oxygen partial pressure, and capillary density had beneficial results with the use of electrical stimulation. This study was retrospective in nature. The patients selected for evaluation met very strict criteria and were divided into treatment groups that were inviolable. They were individually followed at a single clinic, so that even though the overall inter clinic protocol was the same, in each patient's case it did not vary at all. The study population was large enough in each group to give significant data as to efficacy results in preventing recurrence. The overall efficacy among the clinics was superior to that reported in the literature. Mechanical modalities each contributed towards further lowering recurrence rates. SAW and CUSEFS were significantly better than the other modalities at preventing recurrence. The reasoning behind this is that these therapy's both capture more of the healing properties of these modalities, as well as giving them in a more significant and efficacious protocol. SAW has a focal depth of approximately $4 \mathrm{~cm}$. This means that almost all of the acoustic energy is localized to the tissue involved in wound healing. The effect is maximized and the healing that occurs is stronger and thus there is less recurrence. CUSEFS utilizes two physical modalities and they each have an effect both on the tissue and on each other. When the electric wave affects the acoustic wave, it alters it in a way that the energy is refocused at a different angle and along a plane in the tissue

that concentrates the energy more specifically. Ultrasound Assisted Debridement showed the least efficacy among the physical modalities evaluated. While acoustic energy is applied to the liquid debriding medium, much of the energy is dampened by the air before contact with the tissue. Centrifugal debridement is very effective, less painful than sharp debridement, and more exact, but adding Ultrasound does not seem to have a vast effect. The major drawback to this study is that it was retrospective. A prospective study evaluating both the healing rates, efficacy of closure, and then evaluating the recurrence rates would have been preferred.

\section{Conclusion}

Although further research is necessary, it is clear that physical modalities have a positive effect on preventing wound recurrence. Ultrasound both as Surface Acoustic Waves and when combined with Electric Field Stimulation have the most efficacy, while Ultrasound Assisted Debridement has the least effect above Advanced Wound Care Products. A prospective, randomized controlled trial would provide further evidence to this phenomenon.

\section{References}

1. Martins-Mendes D, Monteiro-Soares M, Boyko E, Ribeiro M, Barata P, et al (2014) The independent contribution of diabetic foot ulcer on lower extremity amputation and mortality risk. J Diabetes Complications 28: 632-638.

2. O'Brien J, Finlayson K, Kerr G, Edwards H (2014) The perspectives of adults with venous leg ulcers on exercise: an exploratory study. J Wound Care 23 : 496-468.

3. Dorresteijn J, Kriegsman D, Assendelft W, Valk G (2014) Patient education for preventing diabetic foot ulceration. Cochrane Database Syst Rev 12: CD001488.

4. Waaijman R, De Haart M, Arts ML, Wever D, Verlouw AJ, et al. (2014) Risk factors for plantar foor ulcer recurrence in neuropathic diabetic patients. Diabetes Care 37: 1697-1705.

5. Peters E, Armstrong D, Lavery L (2007) Risk factors for recurrent diabetic foot ulcers. Diabetes Care 30: 2077-2079.

6. Dubsky M, Jirkovska A, Bem R, Fejfarova V, Skibova J, et al. (2013) Risk factors for recurrence of diabetic foot ulcers: prospective follow-up analysis in the Eurodiale subgroup. Int Wound J 10: 555-561.

7. Ghanassia E, Villon L, Thuan dit Dieudonne J, Boegner C, Avignon A, et al 
Citation: Rosenblum J, Gazes MI, Rosenblum S, Karpf A, Greenberg N (2017) A Multicenter Evaluation of Chronic Ulcer Recurrence with the Use of Varying Mechanical Wound Healing Modalities. J Vasc Med Surg 5: 323. doi: 10.4172/2329-6925.1000323

(2008) Long-term outcome and disability of diabetic patients hospitalized for diabetic foot ulcers. Diabetes Care 31: 1288-1292.

8. Apelqvist J, Larsson J, Agardh CD (1993) Long-term prognosis for diabetic patients with foot ulcers. J Intern Med 233: 485-491.

9. Galea A, Springett K, Bungay H, Clift S, Fava S (2009) Incidence and location of diabetic foot ulcer recurrence. The Diabetic Foot Journal 12: 181-186.

10. Lavery L, Higgins K, Lanctot D, Constantinides G, Zamorano R (2007) Preventing diabetic foot ulcer recurrence in high-risk patients: use of temperature monitoring as a self-assessment tool. Diabetes Car 30: 114-120.

11. Collins L, Seraj S (2010) Diagnosis and Treatment of Venous Ulcers. Am Fam Physician 81: 989-996.

12. Nelson E, Bell-Syer S, Cullum N (2000) Compression for preventing recurrence of venous ulcers. Cochrane Database Syst Rev 4: CD002303.

13. McDaniel H, Marston W, Farber M, Mendes R, Owens L (2002) Recurrence of chronic venous ulcers on the basis of clinical etiologic anatomic and pathophysiologic criteria and air plethysmography. J Vasc Surg 35: 723-728.

14. Reeder S, Eggen C, Maessen-Visch M, De Roos K, Neumann H (2013) Recurrence of venous leg ulceration. Reviews in Vascular Medicine 1: 63-65.

15. Dolibog P, Franek A, Taradaj J, Blaszczak E, Cierpka L (2008) Efficiency of Therapeutic Ultrasound for Healing Venous Leg Ulcers in Surgically-treated Patients.
16. Yao M, Hasturk H, Kantarci A, Gu G, Garcia-Lavin S, et al. (2012) A pilot study evaluating noncontact low frequency ultrasound and underlying molecular mechanism of diabetic foot ulcers. Int Wound J.

17. Herberger K, Debus E, Larena-Avellaneda A, Blome C, Augustin M (2012) Effectiveness Tolerability and Safety of Electrical Stimulation of Wounds with an Electrical Stimulation Device: Results of a Retrospective Register Study Wounds 24: 76-84.

18. Peters E, Lavery L, Armstrong D, Fleischli J (2001) Electric stimulation as an adjunct to heal diabetic foot ulcers: a randomized clinical trial. Arch Phys Med Rehabil 82: 721-725

19. Baker L, Chambers R, DeMuth S, Villar F (1997) Effects of electrical stimulation on wound healing in patients with diabetic ulcers. Diabetes ulcers 20: 405-412.

20. Taradaj J, Franek A, Brzezinska-Wcislo L, Cierpka L, Dolibog P, et al. (2008) The use of therapeutic ultrasound in venous leg ulcers: a randomized controlled clinical trial. Phlebology 23: 178-83

21. Samuels J, Weingarten M, Margolis D, Zubkov L, Sunny Y, et al. (2013) Low-frequency $(<100 \mathrm{kHz}$ ) low intensity $(<100 \mathrm{~mW} / \mathrm{cm}(2))$ ultrasound to treat venous ulcers: a human study and in vitro experiments. J Acoust Soc Am 134: 1541-1547.

22. Junger M, Arnold A, Zuder D, Stahl H, Heising S (2008) Local therapy and treatment costs of chronic venous leg ulcers with electric stimulation (Dermapulse): a prospective, placebo controlled double blind trial. Wound Repair Regen 16: 480-487. 